\title{
Charge-doping-induced phase transitions in hydrogenated and fluorinated graphene
}

\author{
Tim O. Wehling* \\ Institute for Theoretical Physics, Universität Bremen, Otto-Hahn-Allee 1, 28359 Bremen, Germany \\ and Bremen Center for Computational Material Science, Universität Bremen, Am Fallturm 1, 28359 Bremen, Germany \\ Bernhard Grundkötter-Stock, Bálint Aradi, and Thomas Frauenheim \\ Bremen Center for Computational Material Science, Universität Bremen, Am Fallturm 1, 28359 Bremen, Germany \\ Thomas Niehaus \\ Department of Theoretical Physics, University of Regensburg, 93040 Regensburg, Germany \\ (Received 9 February 2014; revised manuscript received 7 August 2014; published 20 August 2014)
}

\begin{abstract}
We show that charge doping can induce transitions between three distinct adsorbate phases in hydrogenated and fluorinated graphene. By combining ab initio, approximate density functional theory and tight-binding calculations we identify a transition from islands of $\mathrm{C}_{8} \mathrm{H}_{2}$ and $\mathrm{C}_{8} \mathrm{~F}_{2}$ to random adsorbate distributions around a doping level of $\pm 0.05 \mathrm{e} / \mathrm{C}$ atom. Furthermore, in situations with random adsorbate coverage, charge doping is shown to trigger a sublattice ordering transition when the doping level exceeds the adsorbate concentration. Rehybridization and lattice distortion energies make graphene, which is covalently functionalized from one side only, most susceptible to these two kinds of phase transitions. The energy gains associated with the clustering and ordering transitions can exceed room temperature thermal energies.
\end{abstract}

DOI: 10.1103/PhysRevB.90.085422

PACS number(s): 73.22.Pr, 68.43.Bc, 72.80.Vp, 73.20.Hb

\section{INTRODUCTION}

Low dimensional materials provide unique opportunities to manipulate their properties by chemical means. Graphene in particular is a zero band gap Dirac material which can be turned into a wide band gap insulator by hydrogenation [1] or fluorination [2,3]. Partially functionalized graphene offers a unique chance to tune optical and electronic transport properties between disordered Dirac material [4] and insulating characteristics by varying the adsorbate concentration [1] and the real space arrangement of the adsorbates [3,5-9]. Similarly, electron correlation phenomena including magnetism [10] and superconductivity [11] can be expected to be most sensitive to adsorption patterns in chemically functionalized graphene. It is thus crucial to be able to tune real space arrangements of adsorbates for on-demand functionalization of graphene.

Interestingly, field theoretical studies suggested various structural phase transitions in dilute graphene adsorbate systems including instabilities towards Kekulé and sublattice symmetry broken patterns [7,12-15]. It remained, however, unclear which of these transitions could be realized experimentally, particularly in situations with sizable adsorbate coverage ( $\sim 5 \%$ to $20 \%$ ).

In this paper we show that adsorption patterns of hydrogen and fluorine atoms on graphene can be largely manipulated by charge doping. By combining ab initio density functional theory (DFT), the density functional tight-binding scheme (DFTB), and tight-binding calculations we find that charge doping can induce transitions between phases with homogeneous adsorbate distribution over the entire sample and separation into clean graphene and areas with maximum adsorbate coverage (Fig. 1). We furthermore find that in case of homogeneous adsorbate distribution, charge doping can

\footnotetext{
*wehling@itp.uni-bremen.de
}

trigger an ordering transition where the sublattice symmetry is spontaneously broken.

In general, the interplay of several mechanisms determines the stability of graphene derivatives: First, covalent adsorbates like $\mathrm{H}$ or $\mathrm{F}$ lift their $\mathrm{C}$ bonding partners out of the graphene plane and rehybrizdize them from $s p^{2}$ to $s p^{3}$. There are furthermore electronic energies associated with bond formation as well as electronically mediated interactions between adsorbates [7,12-17]. We show that rehybridization and lattice distortion energies make graphene, which is covalently functionalized from one side only, most susceptible to the above mentioned phase transitions.

The paper is organized as follows: In Sec. II we summarize the quantum mechanical models and methods used to calculate total energies of different functionalized graphene systems. The results of our study are discussed in Sec. III. Readers not interested in technical details might directly jump to Sec. III. Conclusions are given in Sec. IV.

\section{METHODS}

Different level quantum mechanical calculations are combined to obtain the total energies of the graphene adsorbate systems and their doping dependence. First, DFTB calculations were carried out using the DFTB+ program package [18] (version 1.2.2) with the parametrization sets mio-1-1 [19] for $\mathrm{H}$ adsorption and pbc-0-3 [20] for $\mathrm{F}$ adsorption. The doping has been simulated by employing the virtual crystal approach (VCA) [21,22]. For every doping concentration and adsorption type $(X[1: 1]$ and $X[1: 0])$ we considered 20 randomly generated configurations, which have been relaxed until the forces on the atoms were smaller than $10^{-4}$ hartree/bohr. In order to test the reliability of the data, $a b$ initio calculations for a few selected adsorption patterns have been performed using the FHI-AIMS code [23] (version 081912). In these calculations we employed the provided default tight basis sets for the different atom 
(a)

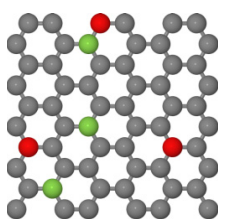

(b)

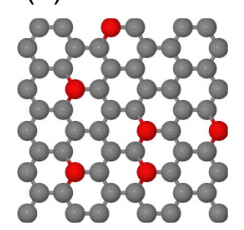

(c)

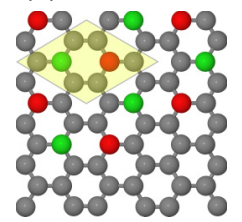

(d)

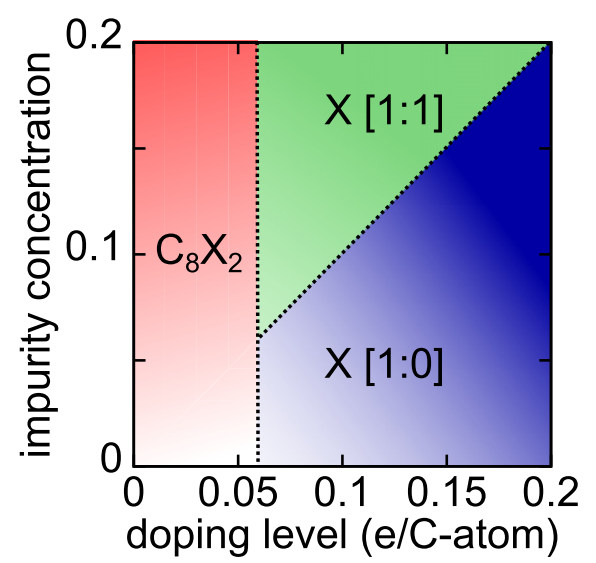

FIG. 1. (Color online) Top views of hydrogen and fluorine adsorption patterns on graphene. Adatoms are colored according to their sublattice position (red-on sublattice A, green-on sublattice B). (a) X[1:1] structure. Fully random coverage with equal population of both sublattices. (b) X[1:0] structure. Hydrogen and fluorine atoms binding randomly to one sublattice only. (c) Phase separation into $\mathrm{C}_{8} \mathrm{X}_{2}$ islands and pristine graphene. The shaded area marks one $\mathrm{C}_{8} \mathrm{X}_{2}$ unit. (d) Schematic illustration of phase diagram of $\mathrm{H}$ and $\mathrm{F}$ adatoms adsorbed to graphene.

types and the PBE exchange correlation functional. The structures were relaxed using similar force criteria as used in DFTB + and the supercell Brillouin zone was sampled on $2 \times 2 \times 1$ Monkhorst-Pack meshes. Indeed, these DFT calculations confirm the DFTB results on variations of the adsorption energies between different patterns as well as trends with doping as shown in Appendix A.

Pure band structure energy differences between different adsorbate phases can be described in terms of a tight-binding model

$$
H=H_{\mathrm{gr}}+H_{\mathrm{imp}}
$$

Here $H_{\mathrm{gr}}=-t \sum_{i, j} c_{i}^{\dagger} c_{j}$ is the nearest-neighbor tight-binding Hamiltonian of graphene, where $c_{i}^{\dagger}\left(c_{i}\right)$ creates (annihilates) an electron at site $i$ and $t=2.6 \mathrm{eV}$ is the hopping parameter. The adsorbates are taken into account through the Hamiltonian

$$
H_{\mathrm{imp}}=\epsilon_{d} \sum_{i^{\prime}} d_{i^{\prime}}^{\dagger} d_{i^{\prime}}+V \sum_{i^{\prime}}\left(d_{i^{\prime}}^{\dagger} c_{i^{\prime}}+\text { H.c. }\right) \text {, }
$$

where $d_{i^{\prime}}^{\dagger}\left(d_{i^{\prime}}\right)$ creates (annihilates) an electron in a defect orbital at a site with adsorbant $\left(i^{\prime}\right)$. The sum runs over all defect sites. The parameters $V=6 \mathrm{eV}, \epsilon_{d}=0$ for hydrogen and $V=$ $6 \mathrm{eV}, \epsilon_{d}=-2 \mathrm{eV}$ for fluorine have been fitted to our DFTB results and are in line with previous DFT calculations [24]. The TB simulations were performed on supercells containing $1800 \mathrm{C}$ atoms and are averaged over 100 impurity configurations each. A comparison of the TB and DFTB total energies is given in Appendix B and shows that energy differences between the adsorbate phases are captured qualitatively correct by the TB model.

\section{RESULTS AND DISCUSSION}

To study the influence of electron and hole doping on adsorption patterns of hydrogen and fluorine on graphene, we have investigated their stability by quantum mechanical simulations. The dependence of adsorption energies $E_{\text {ads }}$ on adsorption patterns and charge doping has been calculated according to

$$
E_{\mathrm{ads}}=\frac{E_{\mathrm{G}: \mathrm{X}}-E_{\mathrm{G}}}{n_{\mathrm{X}}}-\frac{1}{2} E_{\mathrm{X}_{2}} .
$$

Here $E_{\mathrm{G}: \mathrm{X}}$ is the energy of the doped graphene sheet with the adsorbed atoms $\mathrm{X}(\mathrm{X}=\mathrm{H}$ or $\mathrm{F}), E_{\mathrm{G}}$ is the energy of the doped graphene sheet of the same size without the adsorbates, $n_{\mathrm{X}}$ is the number of adatoms, and $E_{\mathrm{X}_{2}}$ is the energy of the adatom dimer. For all random adsorbate distributions considered below, each $E_{\text {ads }}$ presents an average over 20 configurations [25].

First, we consider the adsorption of $\mathrm{H}$ and $\mathrm{F}$ on one side of graphene. Previous DFT calculations showed that the $\mathrm{C}_{8} \mathrm{X}_{2}$ structures [Fig. 1(c) shaded area] correspond to the upper concentration limit for $\mathrm{H} / \mathrm{F}$ single side adsorption $[2,26]$. We thus compare $E_{\text {ads }}$ for the ordered $\mathrm{C}_{8} \mathrm{X}_{2}$ structures to graphene with $10 \%$ adsorbed hydrogen and fluorine adatoms [27] in fully random $\{X[1: 1]$, Fig. 1(a) $\}$ and fully sublattice polarized but otherwise random $\{\mathrm{X}[1: 0]$, Fig. 1(b) $\}$ adsorption patterns in Fig. 2. At zero doping the $\mathrm{C}_{8} \mathrm{X}_{2}$ structures are by several $100 \mathrm{meV}$ per atom more favorable than the $\mathrm{X}[1: 1]$ or $\mathrm{X}[1: 0]$ structures. This finding is in line with the tendency of $\mathrm{H}$ and $\mathrm{F}$ to aggregate when adsorbed on graphene [2,26,28].
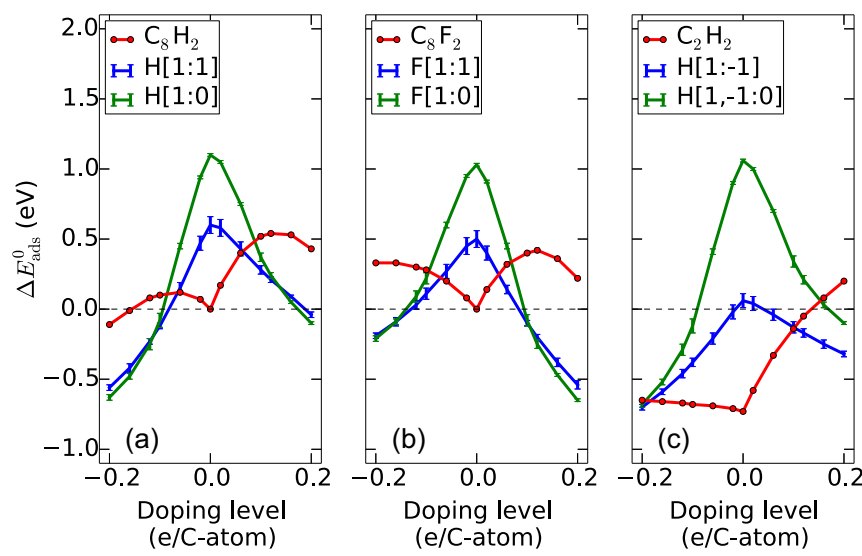

FIG. 2. (Color online) Average relative binding energies (per adatom) for (a) and (c) hydrogen and (b) fluorine adatoms on graphene as function of charge doping for different adsorbate arrangements. (a) and (b) Single sided functionalization. (c) Double sided hydrogenation. Energies are given with respect to the adsorption energy of (a) and (c) $\mathrm{C}_{8} \mathrm{H}_{2}$ and (b) $\mathrm{C}_{8} \mathrm{~F}_{2}$ at zero doping. For the structure with random adsorbate distribution, each data point in (a), (b), and (c) refers to an average over 20 different adsorption configurations with 20 hydrogen/fluorine atoms on a $(10 \times 10)$ graphene cell $(200$ carbon atoms). Error bars give the standard deviations. 
The situation changes, however, drastically with charge doping. With increased doping level the adsorption energy decreases for the quasirandom $\mathrm{X}[1: 1]$ and $\mathrm{X}$ [1:0] patterns but not for the $\mathrm{C}_{8} \mathrm{X}_{2}$ structures. Therefore, carbon-adatom bonds are strengthened in the $\mathrm{X}[1: 1]$ and $\mathrm{X}[1: 0]$ patterns according to the sign convention of Eq. (3). At doping levels above $\pm 0.05 e / \mathrm{C}$ atom the $\mathrm{X}[1: 1]$ configurations become more favorable than $\mathrm{C}_{8} \mathrm{X}_{2}$, with adsorption energy differences exceeding several $100 \mathrm{meV} / \mathrm{X}$ atom. For $\mathrm{H}$ and $\mathrm{F}$ coverage on the order of $10 \%$, charge doping can thus destabilize the separation into clean graphene and $\mathrm{C}_{8} \mathrm{X}_{2}$ islands. Entropic contributions to the free energy could further assist this destabilization of $\mathrm{C}_{8} \mathrm{H}_{2}$ clusters but they turn out to be an order of magnitude smaller than the adsorbate interaction energy gains even at room temperature, as explained in Appendix D. We thus expect a doping driven phase transition from separated $\mathrm{C}_{8} \mathrm{X}_{2}$ islands to disperse adsorbate patterns.

We now turn to situations of doping beyond $\pm 0.05 e / \mathrm{C}$ atom. With increasing charge doping, the adsorption energy difference between sublattice polarized X[1:0] and unpolarized X[1:1] patterns decreases and eventually even reverts sign. The fully sublattice polarized adsorption patterns become lowest in energy at electron and hole doping above $\sim 0.1 \mathrm{e} / \mathrm{C}$ atom for $10 \%$ hydrogen and fluorine coverage, respectively [Figs. 2(a) and 2(b)]. This tendency towards sublattice ordering corresponds to the phase transition suggested in Ref. [7]. Notably, for one-sided adsorption at strong charge doping, we find an energy gain of 60-100 meV/X atom upon sublattice ordering. A comparison of entropic and interaction energy contributions to the free energy [7] shows that sublattice symmetry breaking is expected at temperature $T_{C}=2|\Delta E|$, as soon as the energy gain per adatom $\Delta E$ becomes negative. Therefore, the binding energy differences found here suggest that a second doping-induced phase transition between X[1:1] and X[1:0] structures should be achievable at room temperature and above.

Clearly the electron-hole asymmetry in the doping dependence of adsorption energies [Figs. 2(a) and 2(b)] differs between randomly hydrogenated and fluorinated graphene, which reflects the difference in the polarity of the $\mathrm{C}-\mathrm{X}$ bond.

We furthermore considered hydrogenation from both sides. Here full hydrogenation of graphene, i.e., $100 \%$ hydrogen coverage with $\mathrm{H}$ atoms binding to sublattices $\mathrm{A}$ and $\mathrm{B}$ above and beneath the graphene sheet, respectively, is possible and leads to the formation of graphane [1]. We consider two sided random hydrogen distributions at $10 \%$ coverage (i.e., 5\% above and below), where $\mathrm{H}$ is either sticking to sublattice A only (H[1]:0]) or where it binds to sublattice A from above and B from below $(\mathrm{H}[1: \overline{1}])$. The adsorption energies follow a qualitatively similar trend with charge doping as in the case of single side functionalization [Fig. 2(c)]. With increasing charge doping, hydrogen adsorption energies in the $H[1 \overline{1}: 0]$ and the $H[1: \overline{1}]$ configurations become more negative, while there is an increase in $E_{\text {ads }}$ for graphane with charge doping. However, the formation of graphane is more favorable than random $\mathrm{H}[1 \overline{1}: 0]$ or $\mathrm{H}[1: \overline{1}]$ adsorption patterns over a much wider doping range than in the case of single side hydrogenation. Moreover, there is no doping-induced transition towards a sublattice ordered $\mathrm{H}[1 \overline{1}: 0]$ state in the doping range under investigation. The $\mathrm{H}[1: \overline{1}]$ structure remains more favorable than the $\mathrm{H}[1 \overline{1}: 0]$ state even up to doping levels of $\pm 0.2 \mathrm{e} / \mathrm{C}$ atom. Therefore, doping-induced adatom phase transitions are much easier realized in single side covalently functionalized graphene.

We now aim to identify the microscopic mechanisms behind the charge doping dependent emergence of different adsorbate patterns found above. There are two distinct contributions which determine the dependence of binding energies on doping and adatom patterns: First, adsorbate interactions mediated by the band structure energy of the graphene $\pi$-electron system $[7,12-17]$ and second strain and rehybridization energies. Only the latter contributions distinguish between adatom adsorption from one versus two sides. In the fully sublattice polarized patterns the energy difference between one- and two-sided adsorption is almost an order of magnitude smaller than for patterns with equal sublattice population [cf. Figs. 2(a) and 2(c)]. The rehybridization and strain contributions are thus larger in situations where both sublattices are covered. Previous DFT calculations on graphene with two hydrogen adatoms have shown that binding of two hydrogen atoms to two neighboring $\mathrm{C}$ atoms on different sides of the graphene sheet is by $0.5 \mathrm{eV}$ more favorable than binding on the same side [29]. For $H$ pairs on second or third nearest-neighbor positions the energy differences between single and double side adsorption are at least a factor of 2 smaller. Thus, the large strain and rehybridization energy differences in patterns with coverage of sublattices A and B originate from pairs (or also larger clusters) of hydrogen atoms, which bind to nearest-neighbor carbon atoms.

The band structure energy contribution to the adsorption energy differences can be estimated from the electronic density of states shown for the $\mathrm{C}_{8} \mathrm{H}_{2}, \mathrm{H}[1: 1]$, and $\mathrm{H}[1: 0]$ configurations in Fig. 3. In contrast to the other patterns, the spectrum of $\mathrm{C}_{8} \mathrm{H}_{2}$ exhibits a gap which makes its formation favorable in the undoped case. Due to the gap, charge doping of $\mathrm{C}_{8} \mathrm{H}_{2}$ is however associated with larger band structure energies than for the other two adsorbate configurations. Thus, $\mathrm{C}_{8} \mathrm{H}_{2}$ clusters are destabilized and $\mathrm{H}[1: 1]$ or $\mathrm{H}[1: 0]$ patterns become more favorable at a certain doping level (cf. Fig. 2). The electronic

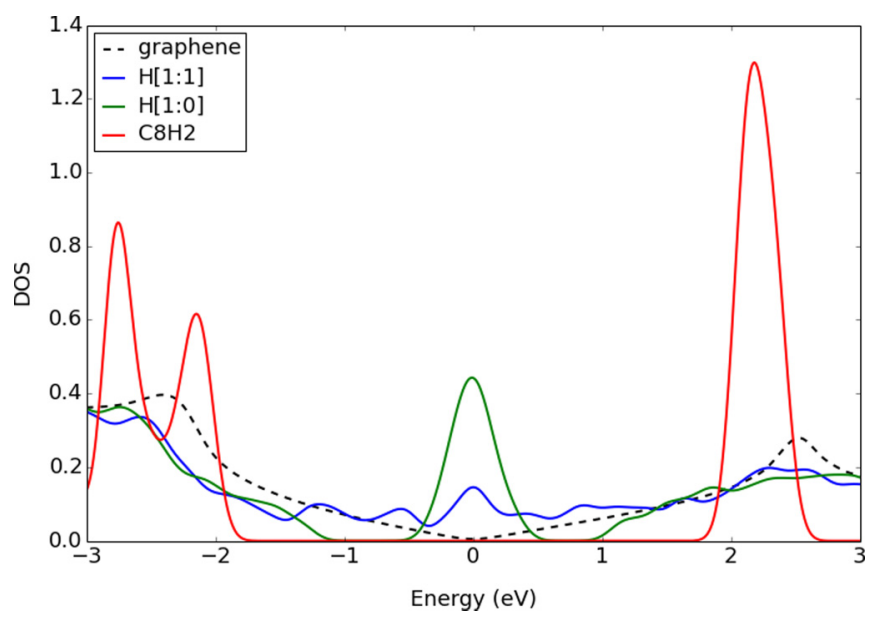

FIG. 3. (Color online) Density of states per unit cell of graphene with different levels of hydrogenation: pristine graphene, $10 \%$ hydrogenation with fully random $\mathrm{H}[1: 1]$ and sublattice ordered $\mathrm{H}[1: 0]$ configuration, and $\mathrm{C}_{8} \mathrm{H}_{2}$. The Fermi level of each configuration has been shifted to zero. 
DOS of the H[1:1] structure is gapless around the Fermi level, while the $\mathrm{H}[1: 0]$ structure exhibits a large peak in the DOS at $E_{F}$. While the large DOS at $E_{F}$ in the undoped state of the $\mathrm{H}[1: 0]$ pattern makes this pattern unstable against structural (as well as possibly magnetic) reconstructions, doping of the $\mathrm{H}[1: 0]$ pattern requires only small amounts of band structure energy. Therefore, the $\mathrm{H}[1: 0]$ pattern becomes eventually most favorable at large doping.

Band structure energy differences can be described in terms of a tight-binding (TB), Eq. (1), which includes the graphene $\pi$ electrons and defect orbitals. We consider the energy difference

$$
\Delta E=E_{\mathrm{ads}}(\mathrm{X}[1: 0])-E_{\mathrm{ads}}(\mathrm{X}[1: 1])
$$

between sublattice polarized $\mathrm{X}[1: 0]$ and unpolarized $\mathrm{X}[1: 1]$ patterns as well as energy differences associated with the phase separation into graphene and $\mathrm{C}_{8} \mathrm{X}_{2}$ islands

$$
E_{\text {sep }}=E_{\mathrm{G}: \mathrm{X}}-\left[(1-4 c) E_{\mathrm{G}}+\frac{1}{2} c n_{\mathrm{C}} E_{\mathrm{C}_{8} \mathrm{X}_{2}}\right],
$$

where $c$ is the adatom coverage and $n_{\mathrm{C}}$ is the number of $\mathrm{C}$ atoms in the supercell. As shown in Appendix B, the TB results significantly deviate from the DFTB for double sided hydrogenation due to strain and rehybridization effects. However, TB turns out to reproduce the DFTB adsorption energy differences for both single side hydrogenation and fluorination qualitatively correct (cf. Fig. 7). The TB model is therefore used to extrapolate the DFTB results and to construct the charge doping and impurity concentration dependent phase diagrams of $\mathrm{H}$ and $\mathrm{F}$ adsorbed to graphene. To this end, we calculated $E_{\text {sep }}$ and $\Delta E$ several concentrations $c$ of adsorbed $\mathrm{H}$ and $\mathrm{F}$, several charge doping levels $n$. We consider the electron doped case only, since the TB model of $\mathrm{H}$ on graphene is particle-hole symmetric and the tendency towards phase transitions in fluorinated graphene is strongest on the electron doped side.

As can be seen from Fig. 4(a), the TB model suggests that the graphene- $\mathrm{C}_{8} \mathrm{H}_{2}$ phase separation occurs at sufficiently small charge doping $(n<0.06 e / \mathrm{C}$ atom $)$ for all adatom concentrations $(c<20 \%)$ considered here. The situation reverts around $n \sim 0.06 \mathrm{e} / \mathrm{C}$ atom, where disperse coverage becomes most favorable. Once the doping exceeds the adatom concentration $(n>c)$, the sublattice symmetry broken $\mathrm{H}[1: 0]$ structures become more favorable than the fully random $\mathrm{H}[1: 1]$ configurations. For fluorine [Figs. 4(b) and 4(d)] a qualitatively similar picture emerges. However, the tendency towards the destruction of the $\mathrm{C}_{8} \mathrm{~F}_{2}$ islands and the sublattice ordering is stronger here.

\section{CONCLUSIONS}

Our DFTB and TB calculations suggest the phase diagram shown in Fig. 1(d). There is a phase separation into graphene and $\mathrm{C}_{8} \mathrm{X}_{2}$ islands at sufficiently small charge doping. For charge doping exceeding $n \sim 0.06 e / \mathrm{C}$ atom the adatoms distribute over the entire sample, where sublattice symmetry breaking becomes favorable when the doping level exceeds the adatom concentration $(n>c)$. In the vicinity of both transition lines more complex phase separated adsorption patterns might emerge. This can be seen from the concave shape of $E_{\text {sep }}$ at $n \sim 0.06 e / \mathrm{C}$ atom [Figs. 4(a) and 4(b)] as well as the steep increase in $\Delta E$ as soon as $c>n$ [Figs. 4(c) and 4(d)].
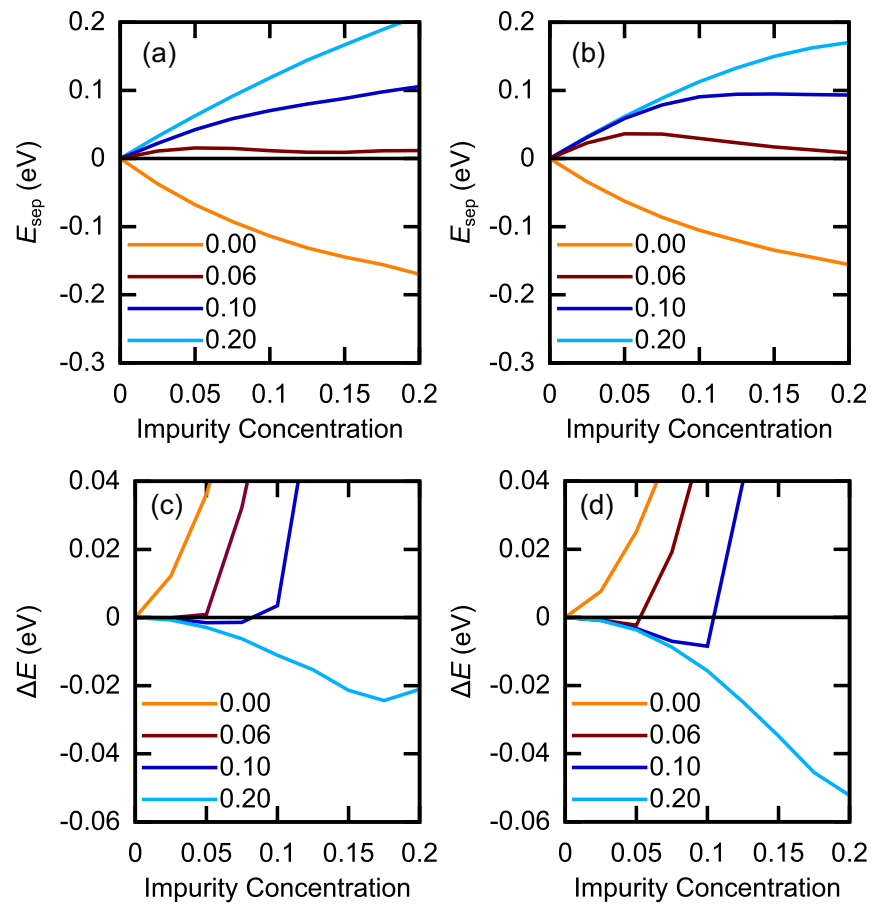

FIG. 4. (Color online) (a) and (b) Energy gains upon phase separation $E_{\text {sep }}$ from fully random $\mathrm{X}[1: 1]$ coverage to $\mathrm{C}_{8} \mathrm{X}_{2}$ islands for single (a) hydrogenated and (b) fluorinated graphene. (c) and (d) Energy gains upon sublattice symmetry breaking $(\mathrm{X}[1: 1] \rightarrow \mathrm{X}[1: 0])$ for (c) hydrogenated and (d) fluorinated graphene. Different curves refer to different charge doping levels between 0 and $0.2 \mathrm{e} / \mathrm{C}$ atom. Energy gains per unit cell are given.

Hydrogen and fluorine adsorption on graphene are highly sensitive to external charge doping. Under which experimental circumstances could switching between different adsorption patterns be expected? Electrostatic doping [30,31] allows us to achieve carrier concentrations on the order of $10^{14} e / \mathrm{cm}^{2}=$ $1 e / \mathrm{nm}^{2} \approx 0.03 e / \mathrm{C}$ atom. According to our results, this alone is not enough to break the tendency towards graphene- $\mathrm{C}_{8} \mathrm{X}_{2}$ phase separation. Chemical doping, for instance, by means of alkali, earth alkali, or rare earth intercalation between graphene and its substrate, however, allows for electron doping up to $\approx 0.1 \mathrm{e} / \mathrm{C}$ atom [32-34]. Thus, intercalated graphene samples are the most promising systems to explore the rich variation of covalently functionalized graphene systems with charge doping. In these electron doped systems particularly, fluorine adatoms are highly susceptible to doping-induced phase transitions.

\section{ACKNOWLEDGMENTS}

We thank the European Graphene Flagship and DFG via SPP 1459 for financial support as well as HLRN (projects hbc00011 and hbp00020) for computer time. T.O.W. thanks Björn Trauzettel and Vladimir Fal'ko for useful discussions as well as KITP Santa Barabara for hospitality, where ideas presented in this work were conceived. 


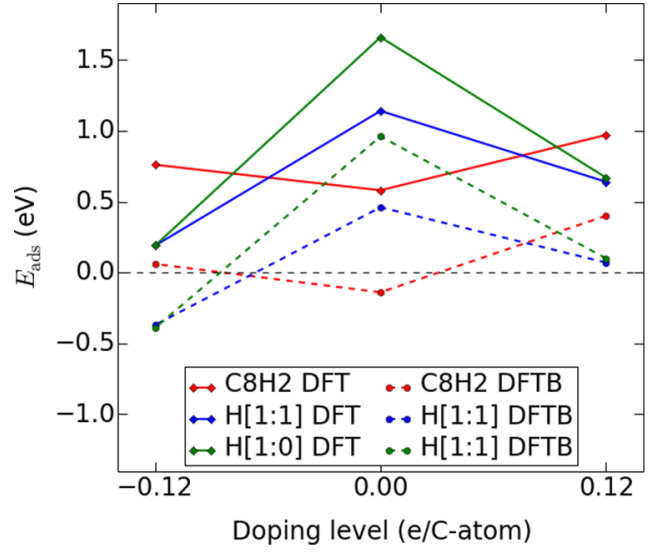

(a)

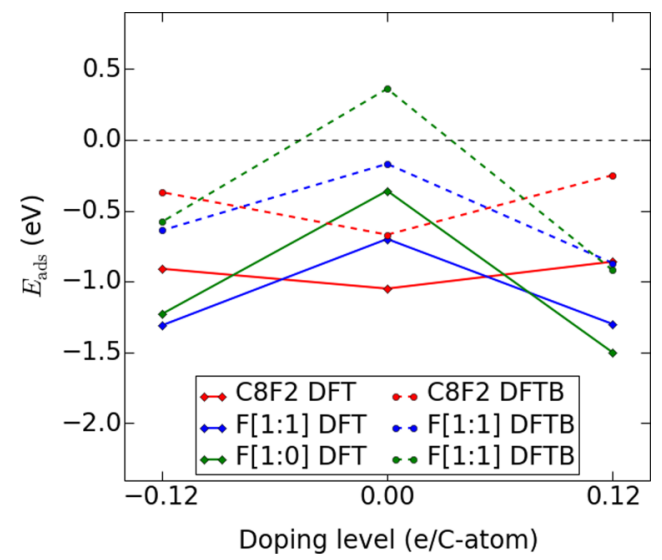

(b)

FIG. 5. (Color online) Adsorption energies for selected (a) hydrogen and (a) fluorine adsorption patterns as calculated by ab initio DFT (solid lines) and by the DFTB methods (dashed lines).

\section{APPENDIX A: COMPARISON OF DFT AND DFTB APPROACHES}

As shown in Fig. 5, the absolute adsorption energies obtained from DFT differ significantly from the DFTB values. $A b$ initio DFT calculations predict the hydrogen adsorption to be unfavorable in the entire investigated doping range, while fluorine adsorption is favorable for all investigated doping levels. It is important to note that the adsorption energies are calculated with respect of pristine graphene and isolated $\mathrm{H}_{2}$ or $\mathrm{F}_{2}$ molecules, which does not resemble typical experimental conditions for hydrogenation and fluorination $[1,3]$.

In contrast to the absolute energies, the relative energies of the various configurations are very similar in both DFTB and DFT. Figure 6 illustrates the relative adsorption energies taking the $\mathrm{C}_{8} \mathrm{X}_{2}$ configuration as reference for each doping level. Both methods predict the $\mathrm{C}_{8} \mathrm{X}_{2}$ configuration being less favorable at doping $\sim \pm 0.1 \mathrm{e} / \mathrm{C}$ atom. Also, both predict that the sublattice polarized configurations become more favorable than the sublattice symmetric ones beyond these doping concentrations.

Indeed, the variations of the adsorption energies between different patterns as obtained from DFTB match the DFT results for hydrogen adsorption almost exactly. In case of fluorine adsorption DFTB with the given parametrization generally overestimates the penalty for building sublattice polarized configurations with respect to sublattice symmetric configurations by approximately $0.2 \mathrm{eV} /$ atom. However, this does not change our statements about the doping dependent phase transition between the sublattice polarized and sublattice unpolarized adsorption patterns qualitatively. These transitions should indeed occur at lower doping concentrations than predicted by the DFTB results.

\section{APPENDIX B: COMPARISON OF TB AND DFTB APPROACHES}

The energy difference (4) between sublattice polarized $\mathrm{X}[1: 0]$ and unpolarized $\mathrm{X}[1: 1]$ patterns as obtained from DFTB and TB model are compared in Figs. 7(a) and 7(b). While the TB results significantly deviate from the DFTB results for double sided hydrogenation, they reproduce the DFTB adsorption energy differences for both single side hydrogenation and fluorination very well. The energy difference $\Delta E$ decreases with charge doping of any sign and changes sign at similar doping levels in DFTB and TB.

We furthermore evaluated the energy difference (5) associated with the phase separation into graphene and $\mathrm{C}_{8} \mathrm{X}_{2}$

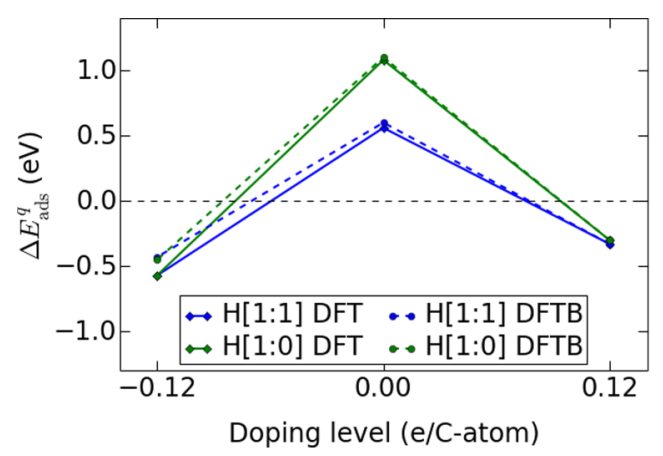

(a)

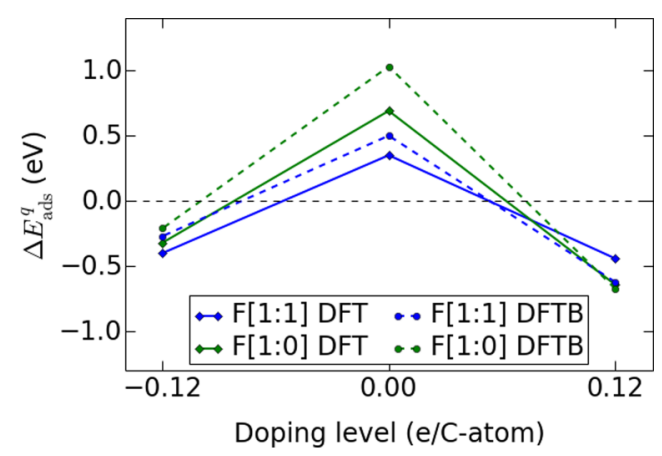

(b)

FIG. 6. (Color online) Adsorption energies relative to the adsorption energy of the (a) $\mathrm{C}_{8} \mathrm{H}_{2}$ and (b) $\mathrm{C}_{8} \mathrm{~F}_{2}$ configurations at various doping levels for selected configurations as calculated by the DFTB method and $a b$ initio DFT method. 

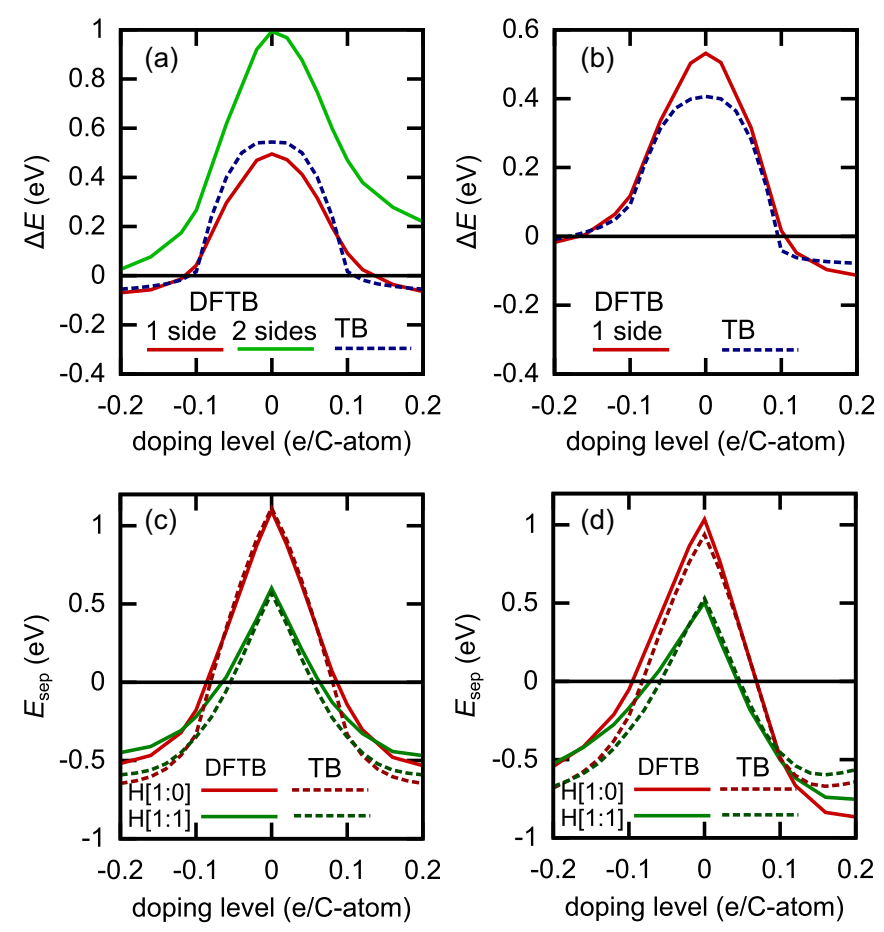

FIG. 7. (Color online) Adsorption energy differences $\Delta E$ [Eq. (4)] between fully random $\mathrm{X}[1: 1]$ and sublattice polarized $\mathrm{X}[1: 0]$ configurations of (a) $\mathrm{H}$ and (b) $\mathrm{F}$ adatoms on graphene. Solid lines are the DFTB results; TB dashed lines. In (a) the adsorption energy difference of hydrogen between sublattice unpolarized $(\mathrm{H}[1: \overline{1}])$ and polarized $(\mathrm{H}[1 \overline{1}: 0])$ patterns is also shown for two side adsorption. (c) and (d) Energy gain $E_{\text {sep }}$ upon phase separation from $\mathrm{X}[1: 0]$ or $\mathrm{X}[1: 1]$ structures to $\mathrm{C}_{8} \mathrm{X}_{2}$ islands. The sample averaged $\mathrm{H} / \mathrm{F}$ coverage is $10 \%$ in all cases.

islands for hydrogenated and fluorinated graphene at $c=10 \%$ coverage and various doping levels [Figs. 7(c) and 7(d)]. There are clearly quantitative differences between $E_{\text {sep }}$ as obtained from the DFTB and the TB model. Nevertheless, the TB models reproduce the doping levels, where the phase separation into graphene and $\mathrm{C}_{8} \mathrm{X}_{2}$ becomes less favorable than the $\mathrm{X}[1: 1]$ or $\mathrm{X}[1: 0]$ configurations, at least qualitatively correct. Also away from this phase transition, energy differences are at least captured qualitatively correct by the TB model.

The TB model is therefore used to extrapolate the DFTB results and to construct the charge doping and impurity concentration dependent phase diagrams of $\mathrm{H}$ and $\mathrm{F}$ adsorbed to graphene. The TB model has furthermore employed estimate finite size effects as well as total energy fluctuations between different disorder realizations as explained below in Appendix C.

\section{APPENDIX C: TOTAL ENERGY FLUCTUATIONS AND DISORDER REALIZATIONS}

The systems under investigation by means of DFTB calculations comprised 200 carbon and 20 impurity atoms. The variance in total energies of the $X[1: 1]$ and $X[1: 0]$ phases (per unit area) depends of course on the simulation cell size and should vanish in the thermodynamic limit (i.e., infinite system size). The standard deviations obtained from 20 disorder realizations are shown as error bars in Fig. 2. These error bars are indeed small as compared to the adsorption energy differences which signal the phase transitions between the cluster and the two disperse phases.

To further study finite size effects manifesting as total energy fluctuations between different disorder realizations we resort to tight-binding simulations which allow us to consider averages of 2000 relations and also to go to systems comprising 800 carbon and 80 impurity atoms. Average energies and standard deviations are summarized in Table I. The histograms summarizing the full spectra of TB data are given in Fig. 8. It becomes clear that the prediction on doping-induced destabilization of the $\mathrm{C}_{8} \mathrm{H}_{2}$ phase is very robust regarding the statistics of data. Energy differences between the two disperse and the $\mathrm{C}_{8} \mathrm{H}_{2}$ cluster phase exceed 10 standard deviations at doping levels $n_{c}=0.0,0.1$, and $0.2 e / \mathrm{C}$ atom. As explained above, sublattice symmetry breaking requires higher doping levels and is thus harder to achieve than the destruction of $\mathrm{C}_{8} \mathrm{H}_{2}$ cluster phase. At sufficiently high doping, however, energy differences between the $\mathrm{X}[1: 1]$ and $\mathrm{X}[1: 0]$ phases exceed the statistical finite size noise from our simulations by three or more standard deviations.

TABLE I. Total energies per adsorbate $E_{\text {sep }}$ and standard deviations $\sigma=\sqrt{\left\langle E_{\text {sep }}^{2}\right\rangle-\left\langle E_{\text {sep }}\right\rangle^{2}}$ at $10 \%$ coverage in the $\mathrm{H}[1: 1]$ and $\mathrm{H}[1: 0]$ phases as obtained from DFTB and TB at different system sizes and doping levels $n_{c}$. The DFTB data refer to 20 disorder realizations, the TB data to 2000 realizations. Energies $E_{\text {sep }}$ are given relative to the $\mathrm{C}_{8} \mathrm{H}_{2}$ cluster phase according to Eq. (5).

\begin{tabular}{lcccccc}
\hline \hline & System size & $n_{c}(e / \mathrm{C}$ atom $)$ & $E_{\text {sep }}(\mathrm{eV})(\mathrm{H}[1: 0])$ & $\sigma(\mathrm{H}[1: 0])$ & $E_{\text {sep }}(\mathrm{eV})(\mathrm{H}[1: 1])$ & $\sigma(\mathrm{H}[1: 1])$ \\
\hline DFTB & 200 & 0.0 & 1.10 & 0.01 & 0.60 & 0.06 \\
TB & 200 & 0.0 & 1.11 & 0.01 & 0.54 & 0.08 \\
TB & 800 & 0.0 & 1.11 & 0.007 & 0.56 & 0.05 \\
DFTB & 200 & 0.1 & -0.14 & 0.03 & -0.23 & 0.03 \\
TB & 200 & 0.1 & -0.34 & 0.01 & -0.34 & 0.03 \\
TB & 800 & 0.2 & -0.34 & 0.007 & -0.35 & 0.02 \\
DFTB & 200 & 0.2 & -0.53 & 0.01 & -0.47 & 0.02 \\
TB & 200 & 0.2 & -0.66 & 0.01 & -0.61 & 0.02 \\
TB & 800 & 0.2 & -0.65 & 0.006 & -0.59 & 0.01 \\
\hline \hline
\end{tabular}



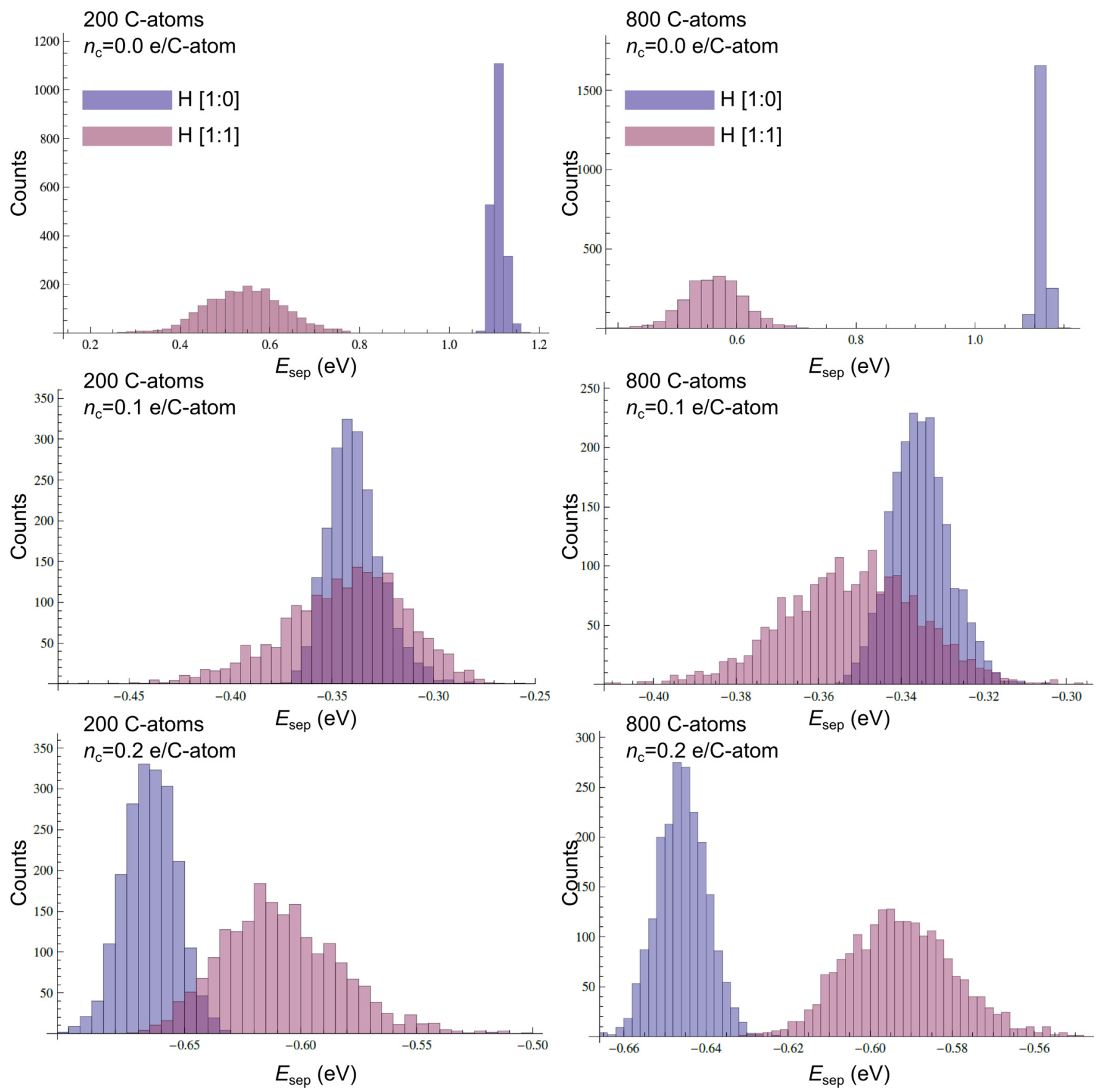

FIG. 8. (Color online) Histograms showing the distributions of binding energy per adatom of the disperse $\mathrm{H}[1: 1]$ and $\mathrm{H}[1: 0]$ with respect to the decomposition into $\mathrm{C}_{8} \mathrm{H}_{2}$ clusters for different doping levels $n_{c}=0.0,0.1$, and $0.2 e / \mathrm{C}$ atom. Data referring to systems comprising $200 \mathrm{C}$ atoms (20 impurity atoms) as well as $800 \mathrm{C}$ atoms ( 80 impurity atoms) are shown. Energies $E_{\text {sep }}$ are given relative to the $\mathrm{C}_{8} \mathrm{H}_{2}$ cluster phase according to Eq. (5).

\section{APPENDIX D: ENTROPY ESTIMATES}

At disperse and random adatom coverage the entropy per site is given by

$$
s(x)=-[x \ln x+(1-x) \ln (1-x)],
$$

where $x$ is the occupation probability of each site. At coverage $c$ (where $c$ is given in terms of impurity atoms per carbon atom), the fully random $\mathrm{X}[1: 1]$ phase has an entropy of
$2 s(c)$ per unit cell, while the $\mathrm{X}[1: 0]$ phase leads to $s(2 c)$ per unit cell. In the $\mathrm{C}_{8} \mathrm{X}_{2}$ phase, the entropy per unit cell vanishes inversely proportional with the cluster size. We discuss here the case of $c=10 \%$ impurity coverage. The entropy gain upon destruction of the $\mathrm{C}_{8} \mathrm{X}_{2}$ phase is thus on the order of $s(0.1) \approx 0.3$. At room temperature $k_{B} T \sim 25 \mathrm{meV}$, entropic contributions $T S \lesssim 10 \mathrm{meV}$ to the free energy $F=E-T S$ are thus an order of magnitude smaller typical energy differences $\left|E_{\text {sep }}\right| \sim 100 \mathrm{meV}$ between the $\mathrm{C}_{8} \mathrm{X}_{2}$ and the X[1:1] phase (cf. Fig. 4). 
[1] D. C. Elias, R. R. Nair, T. M. G. Mohiuddin, S. V. Morozov, P. Blake, M. P. Halsall, A. C. Ferrari, D. W. Boukhvalov, M. I. Katsnelson, A. K. Geim, and K. S. Novoselov, Science 323, 610 (2009).

[2] J. T. Robinson, J. S. Burgess, C. E. Junkermeier, S. C. Badescu, T. L. Reinecke, F. K. Perkins, M. K. Zalalutdniov, J. W. Baldwin, J. C. Culbertson, P. E. Sheehan, and E. S. Snow, Nano Lett. 10, 3001 (2010).

[3] R. Nair, W. Ren, R. Jalil, I. Riaz, V. Kravets, L. Britnell, P. Blake, F. Schedin, A. Mayorov, and S. Yuan, Small 6, 2877 (2010).

[4] T. O. Wehling, A. M. Black-Schaffer, and A. V. Balatsky, arXiv:1405.5774 .

[5] O. Leenaerts, H. Peelaers, A. D. Hernández-Nieves, B. Partoens, and F. M. Peeters, Phys. Rev. B 82, 195436 (2010).

[6] M. Klintenberg, S. Lebègue, M. I. Katsnelson, and O. Eriksson, Phys. Rev. B 81, 085433 (2010).

[7] D. A. Abanin, A. V. Shytov, and L. S. Levitov, Phys. Rev. Lett. 105, 086802 (2010).

[8] J. Schelter, P. M. Ostrovsky, I. V. Gornyi, B. Trauzettel, and M. Titov, Phys. Rev. Lett. 106, 166806 (2011).

[9] H. Sahin, M. Topsakal, and S. Ciraci, Phys. Rev. B 83, 115432 (2011).

[10] R. R. Nair, M. Sepioni, I.-L. Tsai, O. Lehtinen, J. Keinonen, A. V. Krasheninnikov, T. Thomson, A. K. Geim, and I. V. Grigorieva, Nat. Phys. 8, 199 (2012).

[11] G. Savini, A. C. Ferrari, and F. Giustino, Phys. Rev. Lett. 105, 037002 (2010).

[12] A. V. Shytov, D. A. Abanin, and L. S. Levitov, Phys. Rev. Lett. 103, 016806 (2009).

[13] V. V. Cheianov, O. Syljuåsen, B. L. Altshuler, and V. Fal'ko, Phys. Rev. B 80, 233409 (2009).

[14] V. Cheianov, V. Fal'ko, O. Syljuasen, and B. Altshuler, Solid State Commun. 149, 1499 (2009).

[15] V. V. Cheianov, O. Syljuasen, B. L. Altshuler, and V. I. Fal'ko, Europhys. Lett. 89, 56003 (2010).

[16] L. F. Huang, T. F. Cao, P. L. Gong, Z. Zeng, and C. Zhang, Phys. Rev. B 86, 125433 (2012).

[17] D. Solenov, C. Junkermeier, T. L. Reinecke, and K. A. Velizhanin, Phys. Rev. Lett. 111, 115502 (2013).
[18] B. Aradi, B. Hourahine, and T. Frauenheim, J. Phys. Chem. A 111, 5678 (2007).

[19] M. Elstner, D. Porezag, G. Jungnickel, J. Elsner, M. Haugk, T. Frauenheim, S. Suhai, and G. Seifert, Phys. Rev. B 58, 7260 (1998).

[20] C. Köhler and T. Frauenheim, Surf. Sci. 600, 453 (2006).

[21] C. Freysoldt, J. Neugebauer, and C. G. Van de Walle, Phys. Rev. Lett. 102, 016402 (2009).

[22] G. Makov and M. C. Payne, Phys. Rev. B 51, 4014 (1995).

[23] V. Blum, R. Gehrke, F. Hanke, P. Havu, V. Havu, X. Ren, K. Reuter, and M. Scheffler, Comput. Phys. Commun. 180, 2175 (2009).

[24] T. O. Wehling, S. Yuan, A. I. Lichtenstein, A. K. Geim, and M. I. Katsnelson, Phys. Rev. Lett. 105, 056802 (2010).

[25] For a discussion of finite size effects and total energy fluctuations between different disorder realizations see Appendix C.

[26] D. Haberer, C. E. Giusca, Y. Wang, H. Sachdev, A. V. Fedorov, M. Farjam, S. A. Jafari, D. V. Vyalikh, D. Usachov, X. Liu, U. Treske, M. Grobosch, O. Vilkov, V. K. Adamchuk, S. Irle, S. R. P. Silva, M. Knupfer, B. Büchner, and A. Grüneis, Adv. Mater. 23, 4463 (2011).

[27] $100 \%$ adatom coverage refers to one adatom per carbon atom.

[28] L. Hornekær, E. Rauls, W. Xu, Ž. Šljivančanin, R. Otero, I. Stensgaard, E. Lægsgaard, B. Hammer, and F. Besenbacher, Phys. Rev. Lett. 97, 186102 (2006).

[29] D. W. Boukhvalov, M. I. Katsnelson, and A. I. Lichtenstein, Phys. Rev. B 77, 035427 (2008).

[30] C. H. Ahn, J.-M. Triscone, and J. Mannhart, Nature (London) 424, 1015 (2003).

[31] J. Ye, M. F. Craciun, M. Koshino, S. Russo, S. Inoue, H. Yuan, H. Shimotani, A. F. Morpurgo, and Y. Iwasa, Proc. Natl. Acad. Sci. U.S.A. 108, 13002 (2011).

[32] M. S. Dresselhaus and G. Dresselhaus, Adv. Phys. 51, 1 (2002).

[33] J. L. McChesney, A. Bostwick, T. Ohta, T. Seyller, K. Horn, J. González, and E. Rotenberg, Phys. Rev. Lett. 104, 136803 (2010).

[34] D. F. Förster, T. O. Wehling, S. Schumacher, A. Rosch, and T. Michely, New J. Phys. 14, 023022 (2012). 